\title{
Quantum Mechanical Investigation of the Electric and Thermal Characteristics of Magnetic Compound Fluid as a Semiconductor on Metal Combined with Rubber
}

\author{
Kunio Shimada \\ Faculty of Symbiotic Systems Science, Fukushima University, 1 Kanayagwa, Fukushima 960-1296, Japan \\ Correspondence should be addressed to Kunio Shimada, shimadakun@sss.fukushima-u.ac.jp
}

Received 14 February 2011; Accepted 28 March 2011

Academic Editors: L. Y. Khomenkova and K. N. Trohidou

Copyright (C) 2011 Kunio Shimada. This is an open access article distributed under the Creative Commons Attribution License, which permits unrestricted use, distribution, and reproduction in any medium, provided the original work is properly cited.

\begin{abstract}
By applying our developed intelligent fluid, magnetic compound fluid (MCF), to silicon oil rubber, we have made the MCF rubber highly sensitive to temperature and electric conduction. MCF is useful as an element material in haptic robot sensors and other related devices. By mixing metal particles in the silicon oil rubber and by applying a strong magnetic field to the rubber, highdensity clusters of these particles can be formed. In a previous study, we investigated the electric current resulting from the applied voltage. In the present paper, we discuss the capacitance of the MCF rubber. The capacitance as well as the electric current can be explained by quantum theory and behaves as a semiconductor. Regarding the thermal characteristics, in the present paper, the thermal effect on the electric current and the temporary thermal conductivity differ depending on the applied pressure to the MCF rubber and based on the formation of the magnetic clusters. We also explained the tendency of the electric current and the temporary thermal conductivity during the application of heat under low pressure using quantum mechanics theory and clarified the material behavior as a semiconductor based on the thermal characteristics as well as the electric characteristics.
\end{abstract}

\section{Introduction}

Shimada et al. have demonstrated experimentally the use of his intelligent fluid, magnetic compound fluid (MCF) [1-3]. MCF was created by compounding the nm-size magnetite particles $\left(\mathrm{Fe}_{3} \mathrm{O}_{4}\right)$ of magnetic fluid (MF) and the $\mu$ m-size iron $(\mathrm{Fe})$ particles into a single solvent, thereby essentially producing a mixture of MF and magnetorheological fluid (MRF). The apparent viscosity of MCF can be changed by adjusting the composition of the magnetic particles of $\mathrm{Fe}_{3} \mathrm{O}_{4}$ and $\mathrm{Fe}$ and solvent, as discussed by Shimada et al. [1-3] and by Fujita and Shimada [4].We can control the various characteristics of MCF because Shimada succeeded in extracting the magnetic clusters formed by the magnetic particles from both MCF and MRF [5]. In general, the magnetic clusters are constructed of the metal particles involved in the suspensions, MF, MRF, MCF, and so on, and they are called "magnetic clusters" because they have magnetic properties. Since we have come to understand the behavior of the magnetic clusters, MCF has become a candidate for many engineering applications [6]. For example, MCF has been used effectively as a damper in the fields of architecture and vibrating machines [7-9] and for magnetic fluid polishing [10-12]. These applications utilizing MCF have been used in various engineering and industrial fields where damping, polishing, and similar actions are needed, showing that MCF has many engineering applications [6-12].

Rubber with magnetic clusters is a new composite material that responds to a magnetic field, and, by compounding MCF into a silicon oil rubber, the magnetic clusters can be aligned in the rubber by drying the rubber under the application of a magnetic field [13]. The ongoing development of new composite materials is needed in, for example, the fields of robotics, sensing, and related areas, and because the very tensile silicon oil rubber is used in the MCF rubber, the application of this rubber to the artificial skin of robots or to medical instruments is effective. The characteristics of the tension of the MCF rubber have been clarified by Shimada and Zheng [14, 15], as has the relation among electric resistance, Young's modulus, and temperature sensitivity in 
the MCF rubber. These characteristics make MCF rubber applicable for use in a robot's haptic sensors $[16,17]$. By applying the MCF to a silicon oil rubber, we can make MCF rubber highly sensitive to temperature and electric conduction [18-20].

Shimada defined MCF as the suspension of combined magnetic and nonmagnetic metal particles in MF. By mixing $\mathrm{Cu}, \mathrm{Ni}$, or Fe particles and $\mathrm{Fe}_{3} \mathrm{O}_{4}$, the magnetite particles $\mathrm{MF}$, in the silicon oil rubber and by applying a strong magnetic field to it, we can produce magnetic clusters at a high density. We can confirm the formation of the magnetic clusters by extracting them from the suspensions using the following method proposed by Zheng and Shimada [21]. First, a solvent is poured into the fluid, and then the solvent is discarded under the application of a magnetic field induced by a permanent magnet. Thus, the metal and the magnetite particles cannot be drained out. Even if the fluid contains $\mathrm{Cu}$ particles, the nonmagnetic particles of $\mathrm{Cu}$ are trapped in the magnetic clusters and not drained out. This second step of pouring and discarding is repeated many times. Finally, only magnetic clusters remain in the solvent. Thus, we can extract the magnetic clusters from the mixing fluids.

Magnetic clusters are also formed in the mixing rubber by the application of a magnetic field when the rubber is hardening. The particles in the MCF rubber that contains $\mathrm{Cu}$ and $\mathrm{Ni}$ particles and MF form network-like magnetic clusters, which are the same as the clusters from MCF, as shown in Figure 10(a), while the MCF rubber that contains Fe particles and MF forms needle-like magnetic clusters, as shown in Figure 10(b). A comparison between the structures of both types of magnetic cluster shows that the former, with its network-like clusters, has greater temperature and electric sensitivity than does the latter. Thus, by using network-like magnetic clusters in the MCF rubber, we can expect to make a haptic sensor with high electric and temperature sensitivity, as discussed by Zheng and Shimada [22]. The electric conductivity of the MCF rubber that has network-like magnetic clusters is greater than that of ordinary commercial base electric conductive rubber. On the other hand, in the case of the MCF rubber, the electric sensitivity is lower at small deformations but increases at larger deformations, as shown in Figure 11. Therefore, MCF rubber is effective for switching sensors when a small deformation is applied to it. It has high electric conduction sensitivity. Furthermore, the MCF rubber with higher electric sensitivity was improved by condensing Dotite A and B (electric conductivity paints) with carbon blacks (Namamura Rikagaku, Ltd., Co.) [23]. The improved MCF rubber has high resistance to large changes induced by compression. The electric resistance of the improved MCF rubber is megaorder $\Omega$ without compression, and it becomes $335 \Omega$ under $0.294 \mathrm{~N}$ of compression. In general, less than $1 \mathrm{~N}$ is needed for a haptic sensor, for example, for artificial skin.

Using carbon for higher electric resistivity by low force and small displacement is effective, and there have been studies about utilizing carbon blacks [24], carbon fiber [25], or carbon nanotubes [26] in this way. However, in rubber with these carbon materials, the electric resistance without compression is very small, and thus the change of the electric resistivity by compression is also small. In contrast, the improved MCF rubber has greater electric resistivity change because carbon blacks as the other metal particles are involved in the MCF rubber. These facts indicate the differences between MCF rubber and ordinary commercial base electric conductive rubber. Electric current does not flow in the case of touching MCF rubber without any compression force; however, it flows in the case of touching it with large compression force. In contrast, the electric current flows in the case of touching ordinary commercial base electric conductive rubber without any compression force, and it also flows in the case of touching it with large compression force. The change in the flowing electric current is small compared with that in the case of touching it without any compression force. Therefore, the MCF rubber is more effective for switching than is ordinary commercial base electric conductive rubber. The switching effect is more useful for haptic robot sensors because of the larger amount of current flowing in the case of applied large compression force compared with the case of touching it without any compression force. In addition, the MCF rubber is more elastic than the ordinary commercial base electric conductive rubber. In the case of utilizing carbon nanotubes in silicon oil rubber, as an example of ordinary commercial base electric conductive rubber, the resulting rubber is very stiff. It would not be appropriate to use this type of rubber to cover a robot, as the robot would not be able to move easily.

For artificial skin to use on a robot and in other engineering applications, a substance with high sensitivity is needed, and many such substances have been proposed experimentally [27-29]. The substance is usually used by silicon oil rubber. However, the theoretical mechanism of electric conductivity in the rubber with metal particles, with large electric resistivity by compression, has not yet been clarified. The cause is due to that the electric current cannot transit between the metals because of the rubber between the metals by ordinary electric conductive theory. A new theoretical model should be proposed to explain the experimental phenomena that electric current can flow through nonelectric conductive material. A model has been proposed to explain the electric characteristics of ordinary electric conductive rubber, but the theoretical explanation with calculation using quantum theory has not yet been constructed, as discussed by Sherman et al. [30] and by Sheng [31], although the tunnel effect due to the electric conductivity was identified, as well as the potential for the use of this rubber as semiconductor, as discussed by Meijer and Roggen [32]. Shimada [33] clarified theoretically the mechanism of electric conductivity in the rubber with metal particles by using quantum theory, as shown in Appendix C. Conductivity is possible because the electric current flows between the metal particles through the nonmagnetic rubber by the application of voltage because of the tunnel effect.

The theoretical explanation of the mechanism of electric conductivity in rubber with metal particles indicates that this rubber could act as a semiconductor. However, the role of MCF rubber as a semiconductor has not yet been shown. The previous report by Shimada [33] does not include 


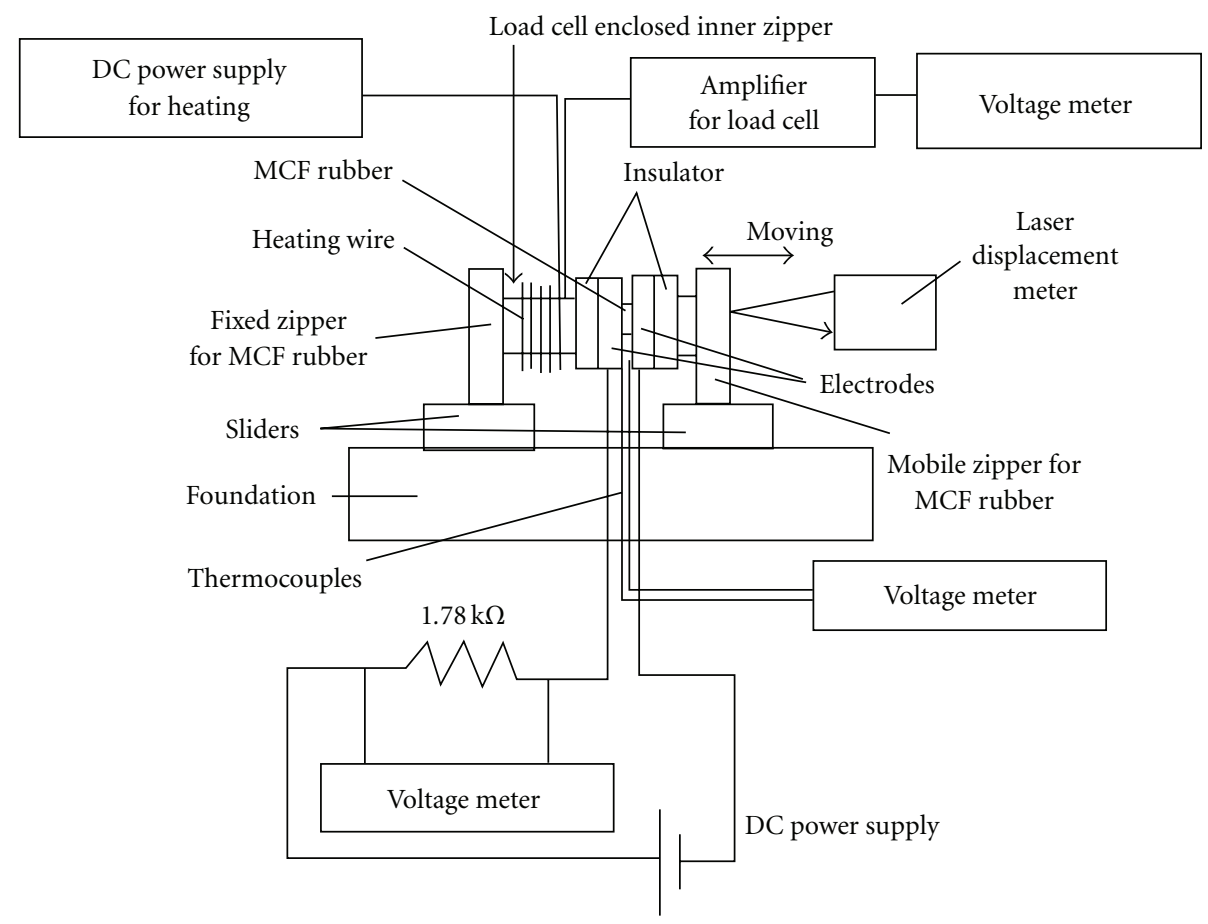

(a)

Details diagram of region around the MCF rubber setted between zippers

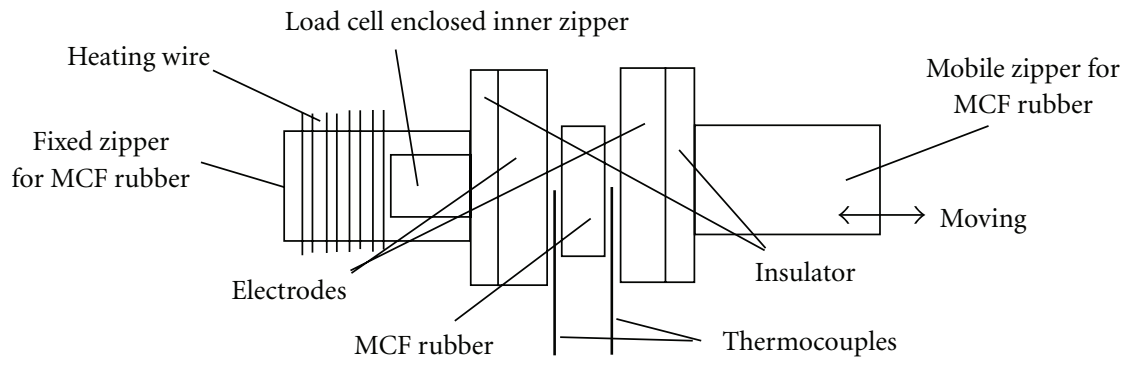

(b)

FIgURE 1: Schematic diagram of our experimental device.

details about the thermal effect on the electric characteristics of MCF rubber and about the investigation of that effect using quantum theory. Therefore, in the present paper, we demonstrate and discuss the use of MCF rubber as a semiconductor.

\section{Experiment Procedure}

We used the same experimental apparatus as was used in a previous study [33], as shown in Figure 1. A load cell (LSM-50K-B, Minebea Co., Ltd., Japan) for measuring the compression force was enclosed by an inner metallic zipper sitting on the slider. This slider was stopped. The zipper was settled at the surface of the nonelectric conductive insulator that was in contact with a pair of metallic electrodes. The MCF rubber was in contact with the opposite end of the electrodes. By moving a second slider, the opposite surface of the MCF rubber was put into contact with another pair of metallic electrodes that was in contact with the other nonelectric conductive insulator. These electrodes could be moved on the slider. The MCF rubber was compressed to make the electrodes move. The displacement of the moving electrodes was measured by a laser instrument (LKG3000VKeyence Co., Ltd., in Japan). In the present study, constant compression force was applied to the MCF rubber, and the electric current was measured when voltage of $10 \mathrm{~V}$, $20 \mathrm{~V}$, or $33 \mathrm{~V}$ (indicated as "input voltage" in the following figures) was applied to the electrodes. Heat was applied to the MCF rubber through the zipper around which the heating wire was wound. The heating was generated as AC with rms values of $70 \mathrm{~V}$ and $1.115 \mathrm{~A}$ (quantity of heat $q=78.0 \mathrm{~W}$ ), $40 \mathrm{~V}$ and $0.655 \mathrm{~A}(q=26.2 \mathrm{~W})$, and $20 \mathrm{~V}$ and $0.335 \mathrm{~A}(q=$ $6.7 \mathrm{~W}$ ), indicated as "heating" in the following figures. The thermocouples were inserted between the MCF rubber and the electrodes, and the temperatures on both surfaces of the MCF rubber were measured. 
We used the same MCF rubber samples as were used in a previous study [33], which were hardened by being placed between nonmagnetic plates with a thickness of $1 \mathrm{~mm}$ and under the application of permanent neodymium magnets with a surface magnetic flux density of $0.56 \mathrm{~T}$. The sample composites were $\mathrm{Ni}: \mathrm{Cu}: \mathrm{MF}$ (HS-50, kerosene base with $50 \mathrm{wt} \%$, Taiho Industrial Co., Ltd., Japan): Dotite A: Dotite B : silicon oil rubber (SH9550, Toray-dow-corning Co., Ltd., Japan) $=1 \mathrm{~g}: 1 \mathrm{~g}: 1 \mathrm{~g}: 1 \mathrm{~g}: 1 \mathrm{~g}: 10 \mathrm{~g}$ to make improved MCF rubber samples, $11.5 \mathrm{~mm} \times 16.2 \mathrm{~mm} \times$ $0.855 \mathrm{~mm}$, and HQ (carbonyl Fe particles, BASF Co., Ltd., Germany) :MF:SH9550 = 6g: $4 \mathrm{~g}: 10 \mathrm{~g}$ samples, $13.1 \mathrm{~mm}$ $\times 18.4 \mathrm{~mm} \times 0.5 \mathrm{~mm}$. The dotites $\mathrm{A}$ and $\mathrm{B}$ are electric conductive glues with carbon powder. We confirmed that the experimental results with the improved MCF rubber were the same as those with the MCF rubber without dotite in the present experiment. Therefore, we show here the results of the MCF rubber with dotite.

A fixed compression force could not be applied by the apparatus shown in Figure 1. Therefore, in the following figures, the quantitative values of the compression force for each test are different. To determine the quantitative tendency at some value not indicated in the figures that is between the values indicated in the figures, one can analogize from the tendency in the figures. This way was confirmed to be correct by many examinations.

\section{Electric Characteristics}

The change in the translated ratio of current $T$ can be calculated by (C.7) in Appendix C, as shown in a previous report [33]. The obtained theoretical results are summarized as follows. The change in the electric current in the case of increasing voltage is qualitatively different from that in the case of decreasing voltage. As applied voltage is increasing, the electric current can be transmitted nonlinearly. The tendency of the electric current to be enhanced gradually with increasing voltage means that the electric resistance is large, because the distance between the metal particles performs as the well-known ordinary space layer confirmed in the case of a semiconductor. Once electric current is transmitted between the metal particles, the route of the transmission of the electric current is up, the electric current can be conducted easily and the electric resistance becomes constant, and then the electric current begins changing to linear. Therefore, when the applied voltage begins decreasing, the tendency of the relation between the voltage and the electric current becomes linear. The electron can be transmitted between the metal particles through the rubber, and it can be transmitted better with more applied voltage, because it has enough energy to get over the energy potential barrier of the rubber. The tendency implies the tunnel effect seen in semiconductors. Therefore, we can conclude that the MCF rubber can act as a semiconductor.

We investigated the capacitance of the MCF rubber by testing it in one of the roles typically filled by a semiconductor. Regarding the MCF rubber as an n-p-type semiconductor, we tried to derive the capacitance of the
MCF rubber from the translated ratio of electric current $T$. As metal particles facing each other can be supposed to be charged the same quantitatively and facing each other as parts of an n-p-type semiconductor, the capacitance between the metal particles facing each other located at $j$ 's turn $C_{j}$ is presented as follows:

$$
C_{j}=\frac{e \varepsilon^{\prime} n_{j} n_{j+1}}{2 \Delta V_{j}\left(n_{j}+n_{j+1}\right)},
$$

where $e$ is the charge of the electron, $\varepsilon^{\prime}$ is the dielectric constant of MCF rubber, $\Delta V_{j}$ is the difference in voltage between the metal particles facing each other, and $n_{j}$ and $n_{j+1}$ are the charges of the metal particles facing each other. As a simple model, if we suppose that the energy arising from the electric current density $J$ does not change velocity but does have a change in the number of electrons, we can estimate the number of electrons caused by heat $n_{e}$ as follows, where $h$ is Planck's constant and $m$ is the mass of the electrons:

$$
n_{j}=J_{j}^{3 / 4}\left(\frac{8 \pi m^{3}}{3 e^{3} h^{3}}\right)^{1 / 4} .
$$

The translated ratio of electric current $T_{j}$ can be presented as follows:

$$
T_{j}=\frac{J_{j+1}}{J_{j}}=\left(\frac{n_{j+1}}{n_{j}}\right)^{4 / 3} .
$$

As the sum of each capacitance between the metal particles facing each other is the capacitance of the MCF rubber, $C$, when the number of segments of the metal particles facing each other in the MCF rubber is $n$, the capacitance $C_{n}$ located at $n$ 's turn can be presented as follows:

$$
\frac{1}{C_{n}}=\sum_{j=1}^{n} \frac{1}{C_{j}} .
$$

From (1)-(4), we can obtain the equation as follows, where $n_{1}$ is the charge on the surface of the MCF rubber and $V$ is the difference in voltage between the surfaces of the MCF rubber:

$$
C_{n}=\frac{e \mathcal{E}^{\prime} n}{2 V \sum_{J=1}^{n}\left(\left(1+T_{j}^{3 / 4}\right) /\left(\prod_{i=1}^{j} T_{i}\right)^{3 / 4} n_{1}\right)} .
$$

$V$ is presented as follows:

$$
\Delta V_{j}=\frac{V}{n} .
$$

The nondimensional expression for capacitance $C$ is presented as follows, where $Q=e n_{1}$ :

$$
C^{*}=\frac{C V}{Q} \text {. }
$$

As a result, the nondimensional capacitance of the MCF rubber, $C_{n}^{*}$, at $n$ 's turn can be presented as follows:

$$
C_{n}^{*}=\frac{\varepsilon^{\prime} n}{2 \sum_{J=1}^{n}\left(\left(1+T_{j}^{3 / 4}\right) /\left(\prod_{i=1}^{j} T_{i}\right)^{3 / 4}\right)} .
$$




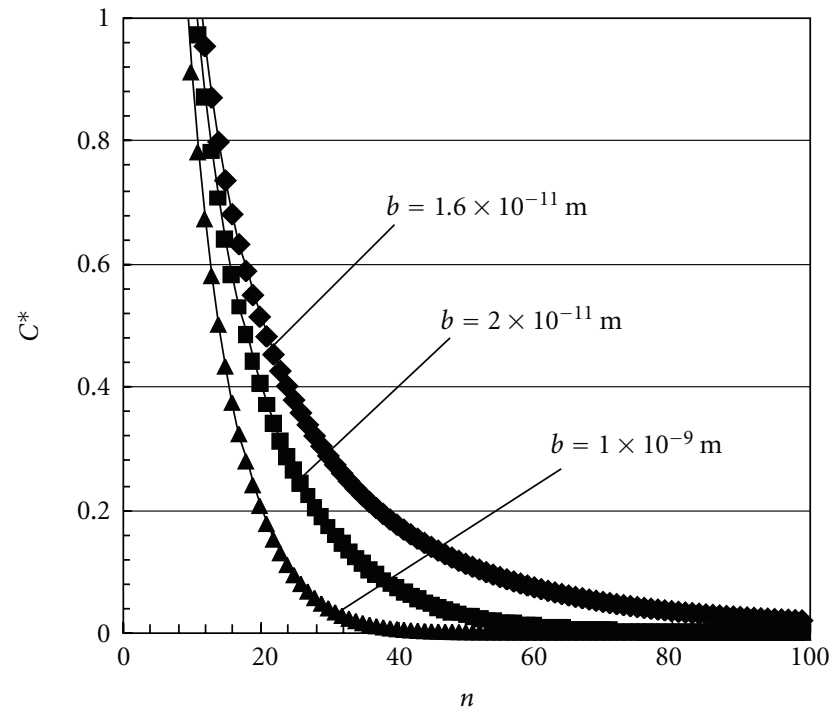

FIgUre 2: Theoretical results of capacitance along the thickness direction of the MCF rubber.

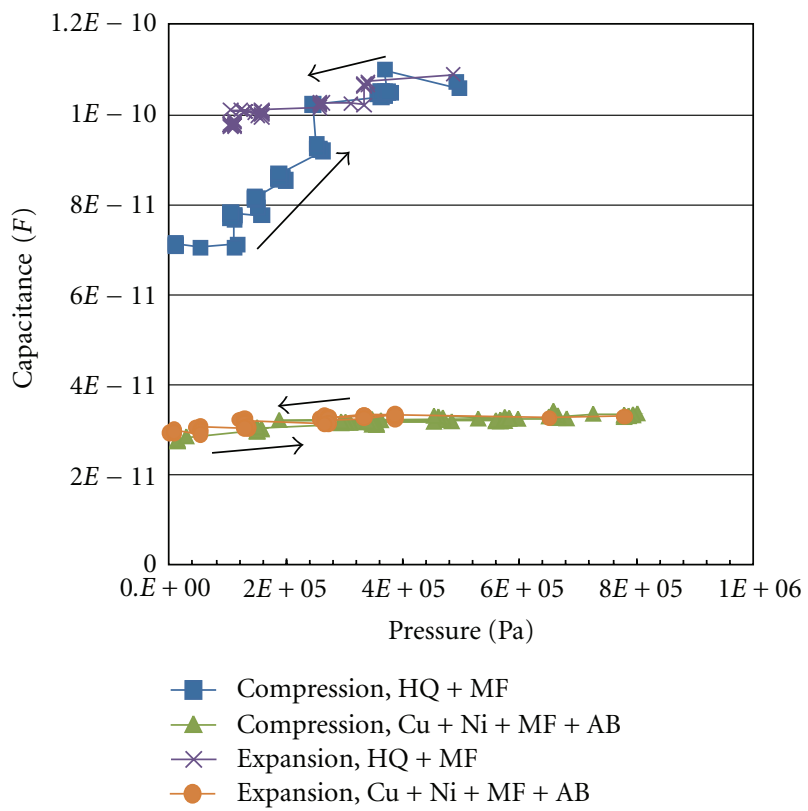

Figure 3: Experimental results of capacitance in response to compression of the MCF rubber.

The theoretical results of the nondimensional capacitance of the MCF rubber, $C_{n}^{*}$, along the points of $n$, that is, along the MCF rubber thickness direction, are shown in Figure 2, where the data is calculated under $V_{o}=4.98 \mathrm{eV}$, $\varepsilon=1.6 \mathrm{eV}, a_{L}=a_{R}=10^{-12} \mathrm{~m}$, and $e E_{o}=0.401 \mathrm{eV}$. When calculating, we used $\varepsilon^{\prime}=0.5$, because we wished to investigate the qualitative tendency. Along the horizontal axis, the electrons are transmitted from the left side to the right side. As the distance across the rubber, $b$, becomes smaller, which means that the MCF rubber is compressed, the capacitance becomes larger.
The experimental results of capacitance of the MCF rubber under compression and expansion are shown in Figure 3. The arrow in the figure indicates the direction of the force from zero to maximum, which means compression, and then from maximum to zero, which means expansion. In this figure and the following figures, $A B$ means Dotites $\mathrm{A}$ and $\mathrm{B}$ in the improved MCF rubber. As the compression increases, the capacitance becomes larger gradually. The results of the present experiment clarify that the relation between the compression force and the compression ratio of the MCF rubber is linear. Therefore, we can confirm that the capacitance becomes larger when the MCF rubber is compressed, which is the same as the tendency shown in the theoretical results. The present quantum theory coincides with the experimental results qualitatively. We can conclude that the rubber combined with metal materials behaves as a semiconductor regarding the capacitance as well as the way in which it handles electric current.

Next, regarding the effect of the magnetic cluster formation, although the qualitative tendencies of the MCF rubber and the improved MCF rubber are the same, we note that the quantitative capacitance of the MCF rubber with $\mathrm{Fe}$ is larger than that of the improved MCF rubber. It was clarified in a previous report [33] that the electric current flows better in the case of the improved MCF rubber formed with network-like magnetic clusters than in the case of the MCF rubber with needle-like magnetic clusters. Therefore, the capacitance of the improved rubber is larger than that of the unimproved rubber.

\section{Thermal Characteristics}

The experimental results of having electric current at compression from the minimum to the maximum amounts in the case of improved MCF rubber are shown in Figure 4. The arrow in this figure and the following figures indicates the compression force from zero to maximum and from maximum to zero. The change in the electric current in the case of increasing compression force is qualitatively different from that in the case of decreasing compression force. This occurs because the distance between the metal particles performs as the well-known ordinary space layer in the case of a semiconductor. The behavior is the same as the relationship between the electric current and the voltage shown in a previous report [33]. In the case of early increasing compression force, as shown the quadrilateral area in the figure, the electric resistance is large, because of the effect of the space layer between the metal particles acting as a semiconductor. The tendency of the relationship of the increasing electric current to the compression force is similar to an exponential curve. As the compression force increases faster, as shown in the quadrilateral area in the figure, the change of the electric current becomes smaller until it reaches a constant level, because the MCF rubber cannot be compressed more and the electric current flows at a constant rate.

Regarding the tendency of the compression force to increase early, as shown in the quadrilateral area in Figure 4, 


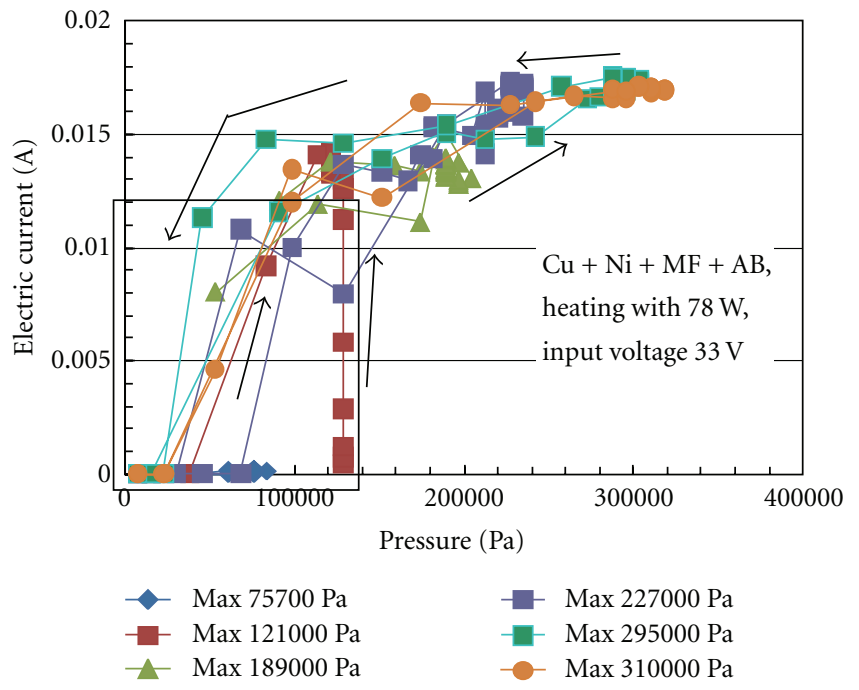

(a)

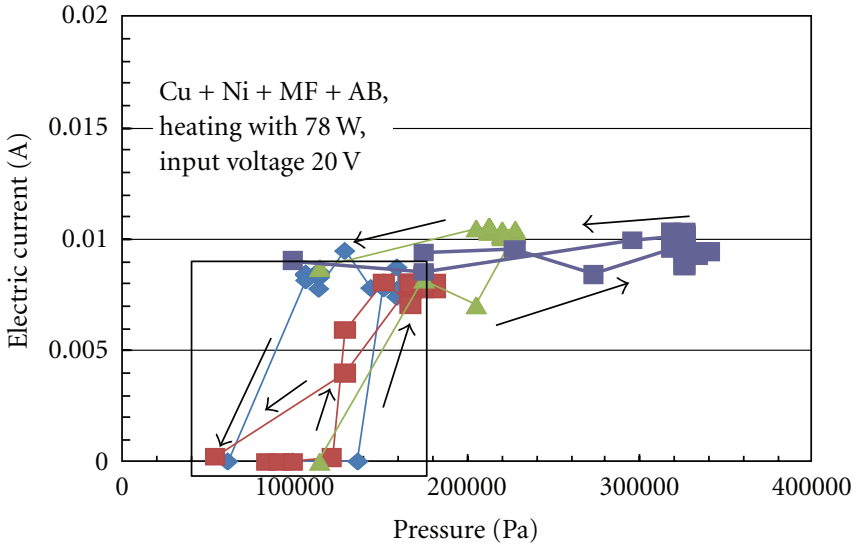

Max $159000 \mathrm{~Pa}$

Max $181000 \mathrm{~Pa}$

- Max $227000 \mathrm{~Pa}$ $\operatorname{Max} 340000 \mathrm{~Pa}$

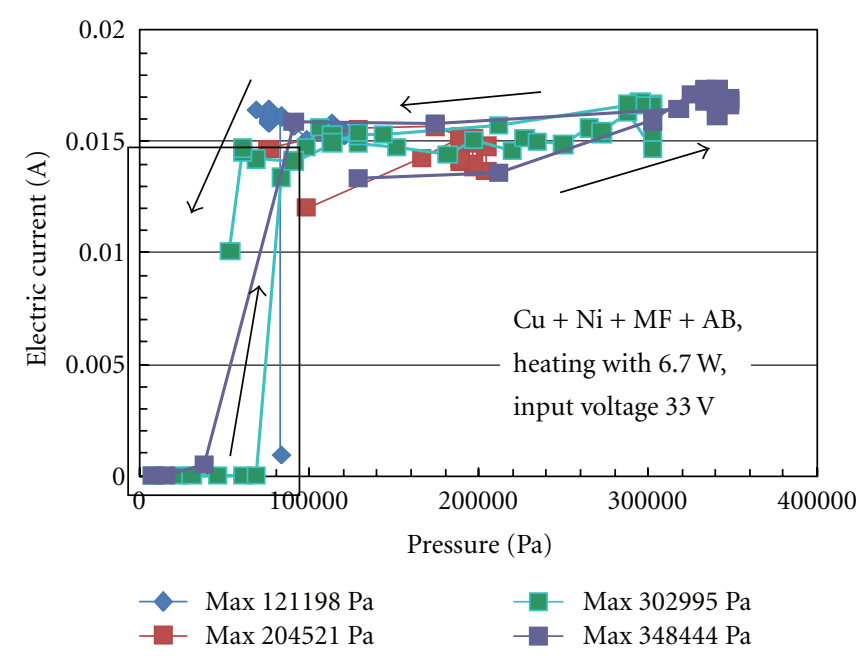

(c)

FIgURE 4: Relation between the electric current and the compression force in the improved MCF rubber.

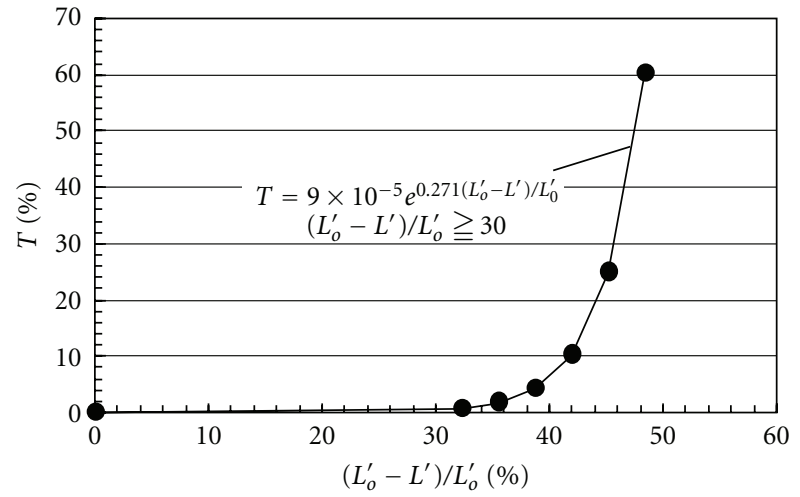

FIGURE 5: Theoretical results of the change in the translated ratio of electric current in relation to the compression ratio of the MCF rubber. the tendency can be explained by the present quantum theory. $T$ is calculated by the equations listed in the previous section under $V_{o}=4.98 \mathrm{eV}, \varepsilon=1.6 \mathrm{eV}, e E_{o}=0.401 \mathrm{eV}$, and $a=10^{-12} \mathrm{~m}$, as shown in Figure 5. $L_{o}^{\prime}$ is the initial thickness of the MCF rubber, and $L^{\prime}$ is the thickness of the MCF rubber at the application of the compression force. As the distance across the rubber, $b$, is smaller, which means that the MCF rubber is compressed, $T$ becomes larger nonlinearly. The increasing tendency is an exponential function. These results are the same even if the voltage and the heat applied to the MCF rubber are changed quantitatively. On the other hand, the relation between the electric current and the compression force in the case of the MCF rubber with Fe particles is that the electric current remains almost constant, as shown in Figure 6. By comparing Figures 6(a) and 6(b) under the same conditions of heating at $26.2 \mathrm{~W}$ and an input voltage of $33 \mathrm{~V}$, we see that the quantitative values of the electric 

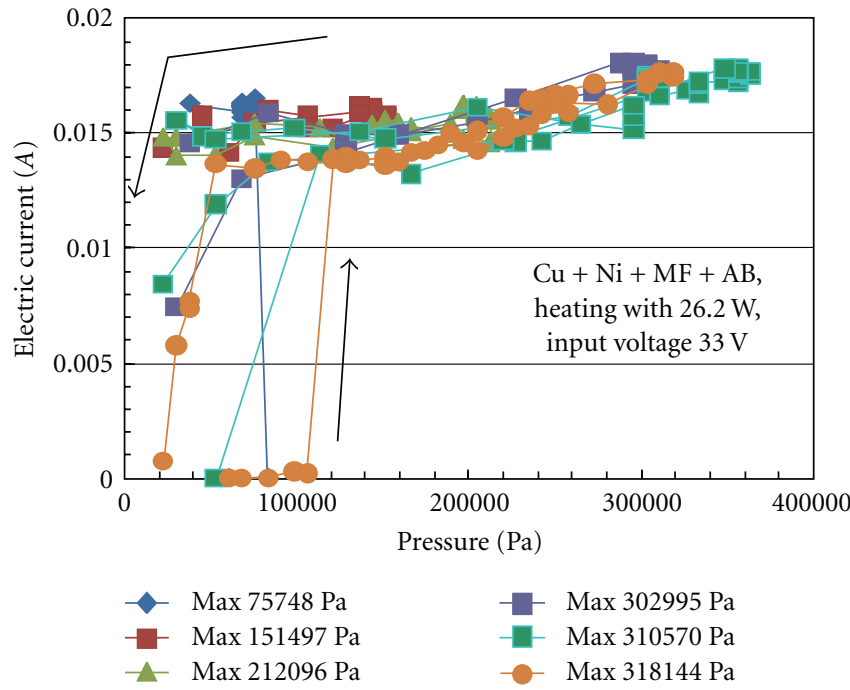

(a)

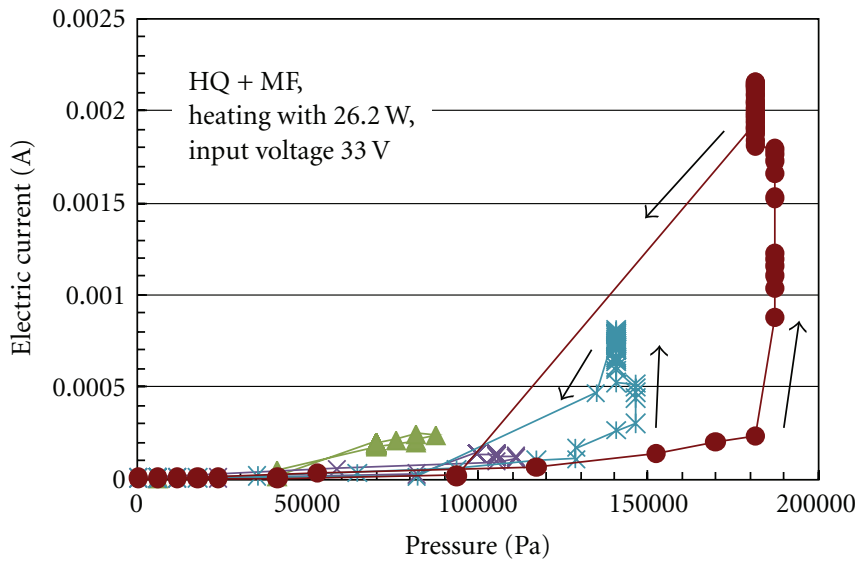

- Max $81964 \mathrm{~Pa}$

* Max 146365Pa

$\lessdot \operatorname{Max} 111238 \mathrm{~Pa}$

(b)

Figure 6: Relation between the electric current and the compression force in the MCF rubber with Fe particles. (a) In the case of improved MCF rubber. (b) In the case of MCF rubber with Fe particles.

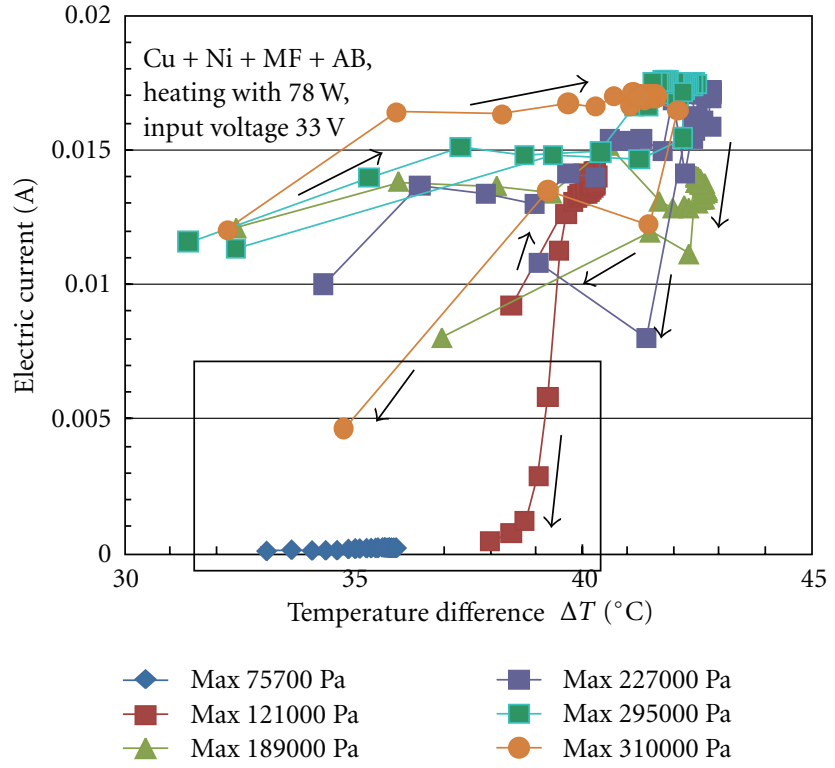

FIGURE 7: Relation of electric current to temperature difference in the improved MCF rubber.

current in the case of Figure 6(b) are smaller than those in the case of Figure 6(a). Because the case shown in Figure 6(b) has needle-like magnetic clusters, the change of the electric current in relation to the compression force is smaller than that in a case with network-like magnetic clusters. This shows the effect of the formation of the magnetic clusters on the electric current in relation to the compression force.

We also investigated the change in the electric current in relation to the temperature difference, $\Delta T$, between the temperatures on both surfaces of the MCF rubber, as shown in Figure 7. In the case of a smaller temperature difference,

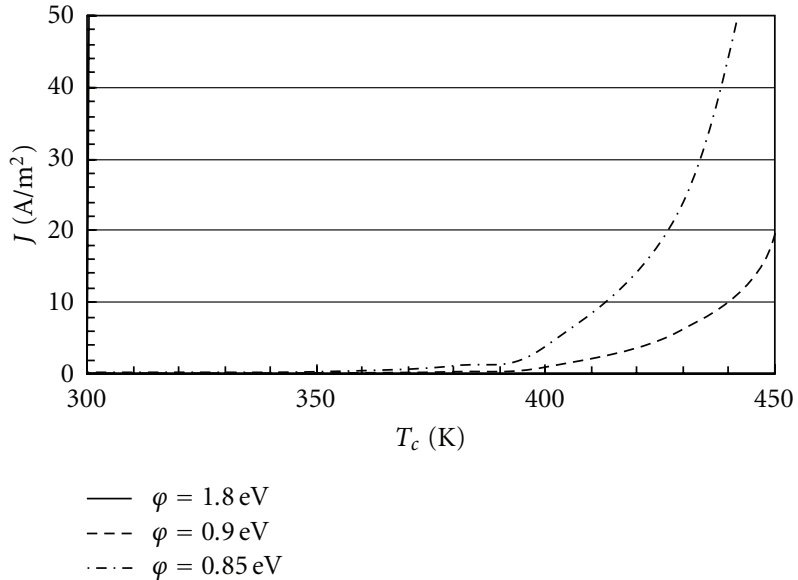

Figure 8: Theoretical results by Richardson's equation as related to quantum theory.

as shown in the quadrilateral area in the figure, the electric current was changed gradually with changing temperature difference. The tendency of the gradually changing electric current is similar to an exponential curve. The tendency can be explained by Richardson's equation as related to quantum theory, as presented by (9), where $T_{c}$ is the temperature at heating, $J$ is the transmitted electric current, $\varphi$ is the work function, and $k$ is the Boltzmann constant:

$$
J=A T_{c}^{2} e^{-\varphi / k T}, \quad A=\frac{4 \pi e m k^{2} T_{c}^{2}}{h^{3}} .
$$

$J$ is presented as the exponential function of $T_{c}$, shown in Figure 8. As the work function can be estimated as the capability of transmitting electrons based on the compression of the MCF rubber, the transmission of the electric current 

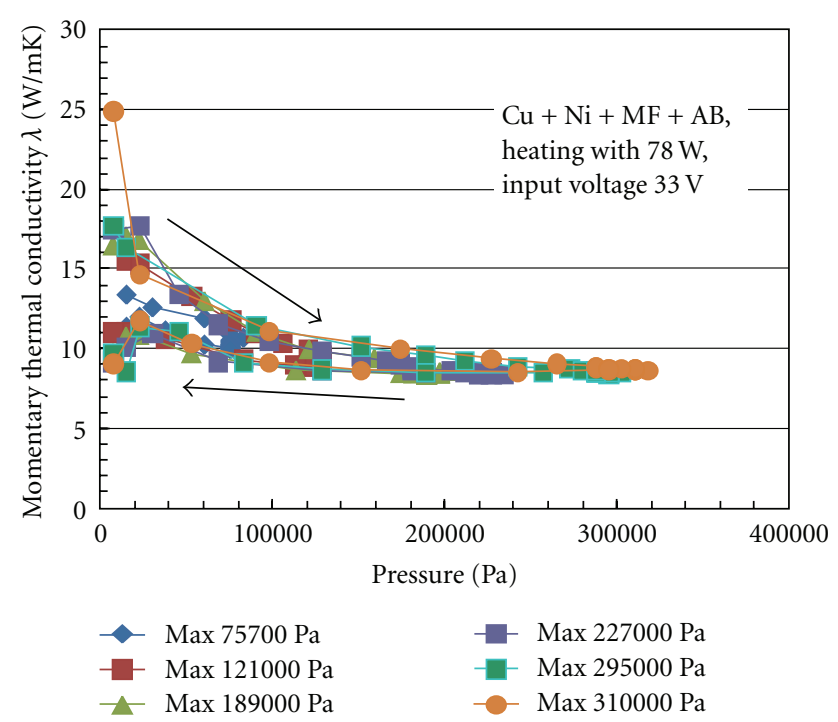

(a)
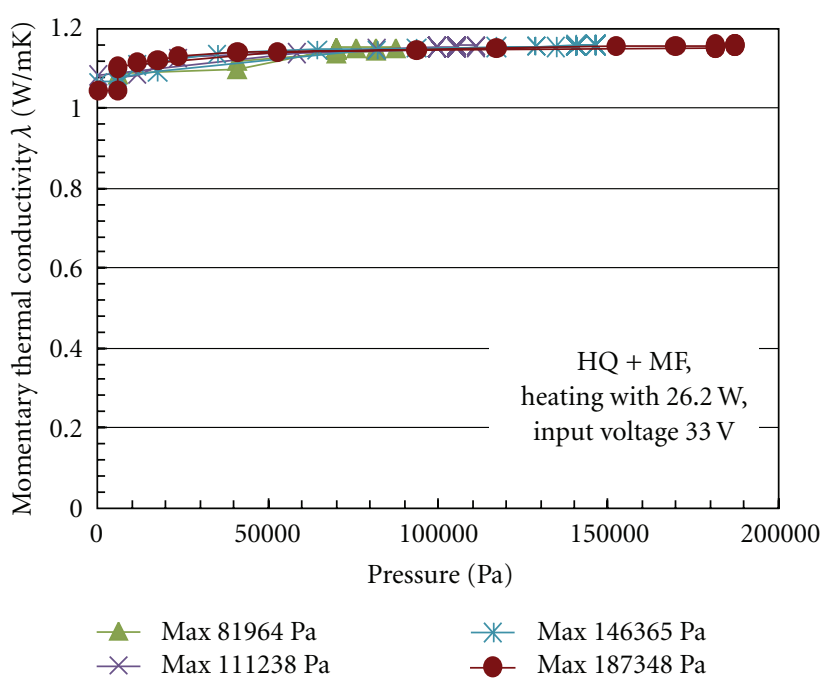

(b)

FIGURE 9: Relation of momentary thermal conductivity to the compression force. (a) In the case of improved MCF rubber. (b) In the case of MCF rubber with Fe particles.

changes grows along with the increased compression of the MCF rubber. The enhancement of heating can be estimated to equal the enhancement of electric current. As a result, with either increased compression force or increased heating, the electric current becomes larger.

Next, to more closely examine the area of large compression force in Figures 4 and 6, we derive the momentary thermal conductivity, $\lambda$, as presented by (10). The thermal conductivity is defined as a momentary value, because the temperature difference and the electric current are changed:

$$
\lambda=\frac{q L_{o}^{\prime}}{\Delta T} .
$$

The relationship of the momentary thermal conductivity to the compression force is shown in Figure 9. In the case of the improved MCF rubber, as the compression force grows, the momentary thermal conductivity becomes smaller. In general, the thermal conductivity of $\mathrm{Ni}$ is $94 \mathrm{~W} / \mathrm{mK}$, that of $\mathrm{Cu}$ is $381 \mathrm{~W} / \mathrm{mK}$, that of carbon is $2.2 \mathrm{~W} / \mathrm{mK}$, that of soft rubber is almost $0.15 \mathrm{~W} / \mathrm{mK}$, and that of kerosene is almost $30 \mathrm{~W} / \mathrm{mK}$. From these values and Figure $9(\mathrm{a})$, we see that the momentary thermal conductivity of the metal particles becomes prevailing at smaller compression force and that of the rubber becomes prevailing at larger compression force. The momentary thermal conductivity dynamically changes under small compression force but is almost constant under large compression force. Thus, we can consider the tendency of the momentary thermal conductivity to be divided into small and large areas of the compression force. In contrast to the improved MCF rubber, in the case of the MCF rubber with Fe particles, the momentary thermal conductivity is almost constant, regardless of the compressed force. The difference in the qualitative tendency between the two types of MCF rubber is due to the difference in the formation of the magnetic clusters. We suspect that the temperature can be transmitted more directly through the MCF rubber than through the complicated magnetic clusters in a network formation.

\section{Conclusion}

The MCF rubber, which contains metal materials, behaves as a semiconductor regarding the capacitance as well as the handling of electric current. The characteristics of the capacitance can be explained by quantum theory and are dependent on the formation of the magnetic clusters. The thermal effect on the electric characteristics of transmitted electric current in the MCF rubber is changed according to the quantitative value of the applied compression force. Especially in the case of a small compression force, the thermal effect on the transmitted electric current can be explained by quantum theory. The tendency is dependent on the formation of the magnetic clusters. Regarding the thermal conductivity of the MCF rubber, in the case of the MCF rubber with network-like magnetic clusters, it reaches a constant at larger compression force. In contrast, in the case of the MCF rubber with needle-like magnetic clusters, it remains almost constant in relation to the compression force.

\section{Appendices}

\section{A. Magnetic Clusters in MCF}

Figure $10(a)$ is a photograph by optical observation of the magnetic clusters extracted from the MCF that contains $\mathrm{Cu}$ particles. The $\mathrm{Cu}$ particles are twig-shaped, with $3 \mathrm{~g}$ and $8-$ $10 \mu \mathrm{m}$ mean length, and the Ni particles are grain-shaped, with $3 \mathrm{~g}$ and $3-7 \mu \mathrm{m}$ mean size in kerosene-based MF with 


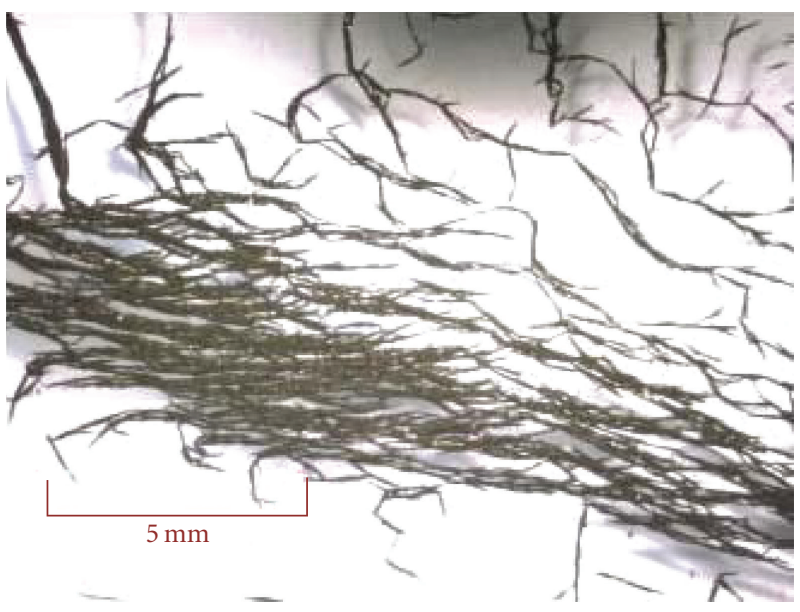

(a)

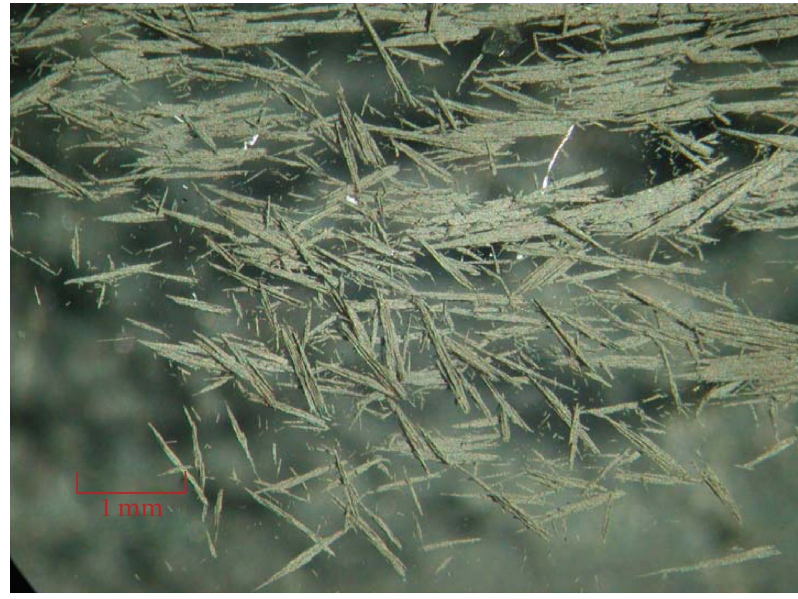

(b)

Figure 10: Photographs of magnetic clusters extracted from MCF. (a) Magnifying ratio of 20 by microscope for magnetic clusters in MCF with $\mathrm{Ni}: 3 \mathrm{~g}, \mathrm{Cu}: 3 \mathrm{~g}, \mathrm{MF}: 4 \mathrm{~g}$. (b) Magnifying ratio of 60 by microscope for magnetic clusters in MCF with Fe: $35 \mathrm{~g}, \mathrm{MF}: 13 \mathrm{~g}$.

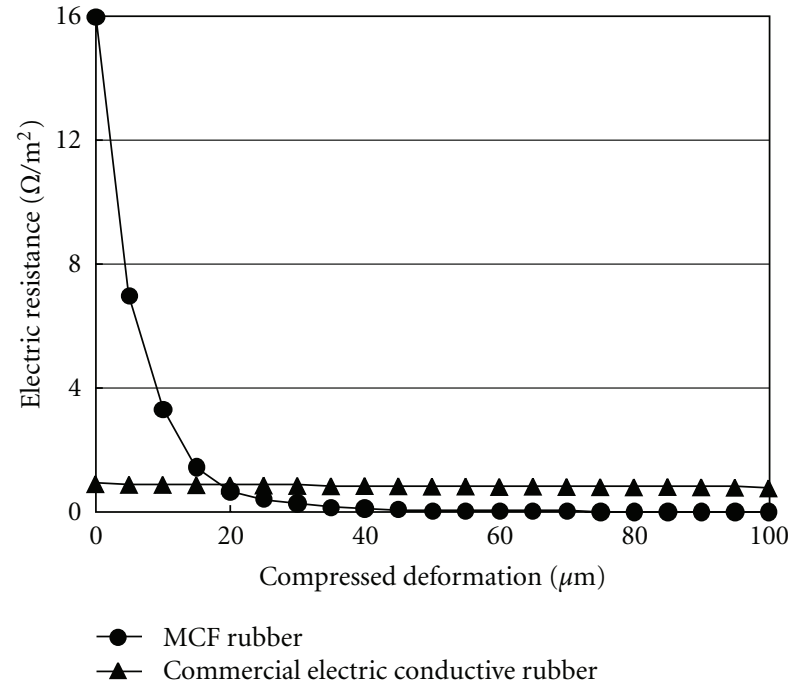

FIGURE 11: Comparison of electric resistance as related to compression deformation between MCF rubber and commercial electric conductive rubber [33].

$4 \mathrm{~g}$ and $50 \mathrm{wt} \%$ of $\mathrm{Fe}_{3} \mathrm{O}_{4}$ that has $10 \mathrm{~nm}$ mean diameter and spherical shape. The magnetic clusters form a network-like shape. On the other hand, Figure 10(b) is a photograph by optical observation of the magnetic clusters extracted from the MCF that contains Fe particles with spheres of $1.2 \mu \mathrm{m}$ mean diameter and $35 \mathrm{~g}$ in kerosene-based MF with $13 \mathrm{~g}$ and $50 \mathrm{wt} \%$ of $\mathrm{Fe}_{3} \mathrm{O}_{4}$. The magnetic clusters form needle-like shapes.

\section{B. Characteristics of Electric Resistance of MCF Rubber}

Figure 11 shows a comparison of electric resistance to compression deformation between MCF rubber and commercial

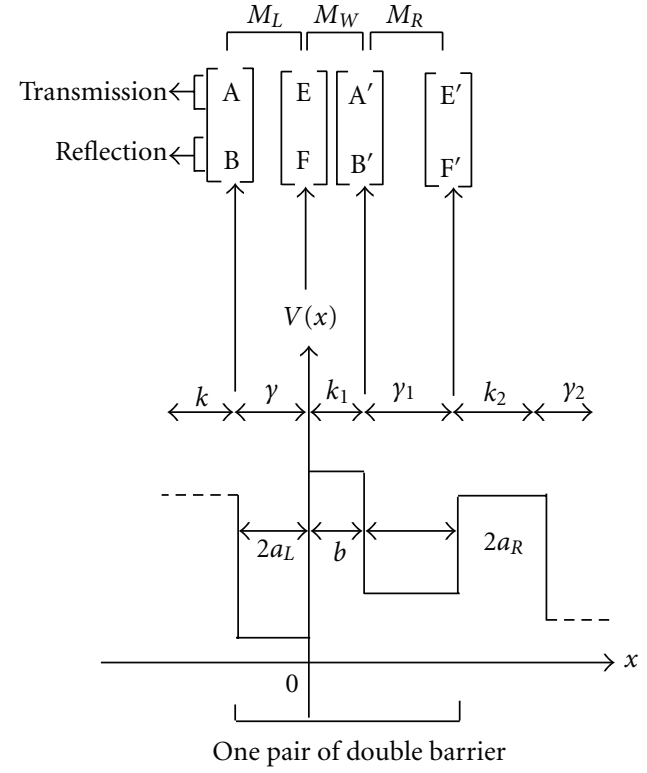

Figure 12: Model of the potential barrier in MCF rubber by quantum theory [33].

electric conductive rubber. The MCF rubber is $20 \mathrm{~mm} \times$ $15 \mathrm{~mm} \times 0.622 \mathrm{~mm}$, and the commercial electric conductive rubber is $30 \mathrm{~mm} \times 30 \mathrm{~mm} \times 0.455 \mathrm{~mm}$.

\section{Quantum Theory on MCF Rubber}

When we examine the electron bond in the metal particles condensing in $\mathrm{MCF}$ rubber, $\mathrm{Fe}_{3} \mathrm{O}_{4}, \mathrm{Cu}, \mathrm{Ni}, \mathrm{Fe}$, and carbon blacks in dotite $\mathrm{A}$ and $\mathrm{B}$, we consider the behavior of the electrons transmitted over the potential barrier of the rubber as Schrödinger's equation one-dimensionally in direction $x$, which is the direction of compression for the MCF rubber and of the transmitting electric current. We can deal with a pair of metal regions of $\gamma$ with a range of $2 a_{L}$ and of $\gamma_{1}$ with 
a range of $2 a_{R}$, and with one rubber region $k$ with a range of $b$ as the tunnel barrier problem on double barriers, and draw the diagram of potential energy $V$ as shown in Figure 12. MCF rubber is organized as $n$ pairs of these two metal and one rubber regions. Wave function $\Psi$ is presented by (C.1), where $\hbar$ is the $h / 2 \pi, V_{o}$ the potential energy at regions $\gamma, \gamma_{1}$, or $k$, and $\varepsilon$ the energy of the electron:

$$
-\frac{\hbar^{2}}{2 m} \frac{\partial^{2} \Psi}{\partial x^{2}}+V_{o}(x) \Psi(x)=\varepsilon \Psi(x) .
$$

From (C.1), $\Psi$ is resolved as (C.2) and (C.3), respectively, for the regions $k$ and $\gamma$, where $A, B, C$, and $D$ are arbitrary constants that can be defined from the continuity of differential coefficients on the boundary between $k$ and $\gamma\left(\gamma_{1}\right)$ regions as a well-known ordinary quantum problem as follows:

$$
\begin{aligned}
& \Psi(x)=A e^{i k x}+B e^{-i k x}, \\
& \Psi(x)=C e^{\gamma x}+D e^{-\gamma x},
\end{aligned}
$$

where

$$
k=\sqrt{\frac{2 m}{\hbar^{2}} \varepsilon}, \quad \gamma=\sqrt{\frac{2 m}{\hbar^{2}}\left(V_{o}-\varepsilon\right)} .
$$

On the one pair of double barriers shown in Figure 12, the arbitrary constants $A$ and $B$ exist in relation to constants $E^{\prime}$ and $F^{\prime}$ as shown in (C.5) as discussed by Roggen and Meijer [34], Roggen and Meijer [35], and Ferry [36], as follows:

$$
\begin{gathered}
{\left[\begin{array}{l}
A \\
B
\end{array}\right]=M_{L} M_{W} M_{R}\left[\begin{array}{l}
E^{\prime} \\
F^{\prime}
\end{array}\right],} \\
M_{T}=M_{L} M_{W} M_{R},
\end{gathered}
$$

where

$$
\begin{aligned}
& M_{L}=\left[\begin{array}{cc}
m_{L 11} e^{i \theta_{L 11}} & m_{L 12} e^{i \theta_{L 12}} \\
m_{L 12} e^{-i \theta_{L 12}} & m_{L 11} e^{-i \theta_{L 11}}
\end{array}\right], \\
& M_{W}=\left[\begin{array}{cc}
e^{-i k_{1} b} & 0 \\
0 & e^{i k_{1} b}
\end{array}\right] \\
& M_{R}=\left[\begin{array}{ll}
m_{R 11} e^{i \theta_{R 11}} & m_{R 21} e^{-i \theta_{R 21}} \\
m_{R 21} e^{i \theta_{L 21}} & m_{R 11} e^{-i \theta_{L 11}}
\end{array}\right], \\
& m_{L 11}=\sqrt{\frac{1}{4}\left(1+\frac{k_{1}}{k}\right)^{2} \cosh ^{2}\left(2 \gamma a_{L}\right)+\frac{1}{4}\left(\frac{k k_{1}-\gamma^{2}}{k \gamma}\right)^{2} \sinh ^{2}\left(2 \gamma a_{L}\right)}, \\
& m_{L 12}=\sqrt{\frac{1}{4}\left(\frac{k k_{1}+\gamma^{2}}{k \gamma}\right)^{2} \sinh ^{2}\left(2 \gamma a_{L}\right)+\frac{1}{4}\left(\frac{k_{1}}{k}-1\right)^{2} \cosh ^{2}\left(2 \gamma a_{L}\right)}, \\
& m_{R 11}=\sqrt{\frac{1}{4}\left(1+\frac{k_{2}}{k_{1}}\right)^{2} \cosh ^{2}\left(2 \gamma_{1} a_{R}\right)+\frac{1}{4}\left(\frac{k_{1} k_{2}-\gamma_{1}^{2}}{k_{1} \gamma_{1}}\right)^{2} \sinh ^{2}\left(2 \gamma_{1} a_{R}\right)}, \\
& m_{R 21}=\sqrt{\frac{1}{4}\left(\frac{k_{1} k_{2}+\gamma_{1}^{2}}{k_{1} \gamma_{1}}\right)^{2} \sinh ^{2}\left(2 \gamma_{1} a_{R}\right)+\frac{1}{4}\left(\frac{k_{2}}{k_{1}}-1\right)^{2} \cosh ^{2}\left(2 \gamma_{1} a_{R}\right)} \text {, } \\
& \theta_{L_{11}}=-\tan ^{-1}\left[\frac{k k_{1}-\gamma^{2}}{\left(k+k_{1}\right) \gamma} \tanh \left(2 \gamma a_{L}\right)\right]+\left(k+k_{1}\right) a_{L}, \\
& \theta_{L_{12}}=-\tan ^{-1}\left[\frac{k k_{1}+\gamma^{2}}{\left(k-k_{1}\right) \gamma} \tanh \left(2 \gamma a_{L}\right)\right]+\pi+\left(k-k_{1}\right) a_{L}, \\
& \theta_{R_{11}}=-\tan ^{-1}\left[\frac{k_{1} k_{2}-\gamma_{1}^{2}}{\left(k_{1}+k_{2}\right) \gamma_{1}} \tanh \left(2 \gamma_{1} a_{R}\right)\right]-\left(k_{1}+k_{2}\right) a_{R}, \\
& \theta_{R_{21}}=\tan ^{-1}\left[\frac{k_{1} k_{2}+\gamma_{1}^{2}}{\left(k_{2}-k_{1}\right) \gamma_{1}} \tanh \left(2 \gamma_{1} a_{R}\right)\right]+\pi+\left(k_{1}-k_{2}\right) a_{R} \text {. }
\end{aligned}
$$


The transmitted probability can be given by

$$
T=\left|M_{T_{11}}\right|^{2} \text {. }
$$

As the present MCF rubber has a lot of metal particles, one pair of double barriers is expanded to $n$ pairs of double barriers as multibarriers. Therefore, the matrix as shown by (C.5) is substituted into the matrix of the neighboring pair of double barriers, and this procedure is repeated to obtain the matrix of $n$ pairs of double barriers, where (C.8) can be given because the material of each region of $k$ and $\gamma$ is the same as that of the other regions. Here, $2 a$ is the thickness of metal particles and $b$ is the thickness of rubber between the metal particles. When $e E_{o}$ is the applied voltage, the voltage $e E$ at the regions of $\gamma$ and $k$ is given as (C.8). Therefore, we calculate (C.5) as substituting $V_{o}-\varepsilon-e E_{o}$ for $V_{o}-\varepsilon$ of $\gamma$ in (C.4):

$$
\begin{gathered}
k=k_{1}=k_{2}=\ldots, \\
\gamma=\gamma_{1}=\gamma_{2}=\ldots, \\
a_{L}=a_{R}=a, \\
e E=\frac{e E o\{(2 n-1) a+2(n-1) b\}}{L^{\prime}} \text { at region of } \gamma \text { before } k, \\
e E=\frac{e E o\{2 n a+(2 n-1) b\}}{L^{\prime}} \text { at region of } \gamma \text { after } k .
\end{gathered}
$$

As the thickness of the MCF rubber is divided into $n$ pairs, the thickness of the MCF rubber $L^{\prime}$ can be given by

$$
L^{\prime}=n \Delta L=2 n(a+b) \quad[m] .
$$

\section{References}

[1] K. Shimada, T. Fujita, H. Oka et al., "Hydrodynamic and magnetized characteristics of MCF (magnetic compound fluid)," Transactions of the Japan Society of Mechanical Engineers, vol. 67, no. 664, pp. 122-128, 2001.

[2] K. Shimada, Y. Akagami, T. Fujita et al., "Characteristics of MCF(Magnetic Compound Fluid)in a rotating rheometer," Journal of Magnetism and Magnetic Materials, vol. 252, pp. 235-237, 2002.

[3] K. Shimada, T. Fujita, S. Kamiyama et al., "Experimental investigation of effects by various factors on shear flow characteristics of magnetic compound fluid (MCF) and its application," Journal of the Japan Society of Applied Electromagnetics and Mechanics, vol. 10, no. 1, pp. 67-72, 2002.

[4] T. Fujita and K. Shimada, "Characteristics and application of magnetorheological fluid," Recent Research Development Magnetism and Magnetic Materials, vol. 1, pp. 463-479, 2003.

[5] K. Shimada, T. Miyazaki, A. Shibayama et al., "Extraction of magnetic clusters self-assembled by a magnetic field," Smart Materials and Structures, vol. 12, no. 2, pp. 297-303, 2003.

[6] K. Shimada, S. Shuchi, H. Kanno et al., "Magnetic cluster and its applications," Journal of Magnetism and Magnetic Materials, vol. 289, pp. 9-12, 2005.

[7] K. Shimada, H. Kanno, J. Ogawa et al., "Development of nonsedimentation type damper," Transactions of the Japan Society of Mechanical Engineers, vol. 69, no. 685, pp. 2075-2082, 2003.
[8] H. Kanno, K. Shimada, and J. Ogawa, "Experimental investigation of variable damping properties for viscous damper utilizing a magnetic responsive fluid," Transactions of the Japan Society of Mechanical Engineers, vol. 71, no. 703, pp. 869-876, 2005.

[9] H. Kanno, J. Ogawa, and K. Shimada, "MR fluid damper composed of different size of particles," International Journal of Applied Electromagnetics in Materials, vol. 25, no. 1-4, pp. 109-112, 2007.

[10] Y. Wu, K. Shimada, Y. C. Wong et al., "Effects of particles blend ratio on surface quality in surface polishing using magnetic polishing liquid (MPL)," Key Engineering Materials, vol. 291292, pp. 337-342, 2005.

[11] K. Shimada, Y. Matuo, K. Yamamoto et al., "Study on new float polishing with the MCF," Journal of Achivements in Materials and Manufacturing Engineering, vol. 23, no. 2, pp. 91-94, 2007.

[12] K. Shimada, Y. Matuo, K. Yamamoto et al., "A new floatpolishing technique with large clearance utilizing magnetic compound fluid," International Journal of Abrasive Technology, vol. 1, no. 3-4, pp. 302-315, 2008.

[13] K. Shimada, S. Shuchi, and H. Kanno, "Magnetic rubber having magnetic clusters composed of metal particles," Journal of Intelligent Material Systems and Structures, vol. 16, pp. 1520, 2005.

[14] K. Shimada and Y. Zheng, "Basic study on tensile characteristics of MCF (magnetic compound fluid) rubber," Transactions of the Japan Society of Mechanical Engineers, vol. 74, no. 747, pp. 2287-2294, 2008.

[15] K. Shimada and Y. Zheng, "Study on electric conductivity under tensile condition of MCF rubber for haptic robot sensor," Transactions of the Japan Society of Mechanical Engineers, vol. 74, no. 744, pp. 1748-1754, 2008.

[16] M. Tanaka, J. Hiraizumi, J. L. Leveque et al., "Haptic sensor for monitoring skin conditions," International Journal of Applied Electromagnetics and Mechanics, vol. 14, pp. 397-404, 2002.

[17] R. Tajima, S. Kagami, M. Inaba, and H. Inoue, "Development of soft and distributed tactile sensors and the application to a humanoid robot," Advanced Robotics, vol. 16, no. 4, pp. 381397, 2002.

[18] K. Shimada and Y. Zheng, "Development of MCF rubber with temperature and electric senses for an elemnt material in haptic robot sensor," Transactions of the Japan Society of Mechanical Engineers, vol. 73, no. 735, pp. 2269-2274, 2007.

[19] Y. Zheng and K. Shimada, "Study on a haptic sensor using MCF (magnetic compound fluid) electric conductive rubber," Journal of Solid Mechanics and Materials Engineering, vol. 2, no. 6, pp. 748-755, 2008.

[20] Y. Zheng, K. Shimada, and Y. Ido, "Research on a haptic sensor made using MCF conductive rubber," Journal of Physics Condensed Matter, vol. 20, no. 20, Article ID 204148, 2008.

[21] K. Shimada, T. Miyazaki, A. Shibayama et al., "Extraction of magnetic particle clusters self-assembled by a magnetic field," Smart Materials and Structures, vol. 12, no. 2, pp. 297-303, 2003.

[22] Y. Zheng and K. Shimada, "Basic study on trial production of haptic sensor utilizing MCF(magnetic compound fluid)electric conductive rubber," Jounal of Japan Society Experimental Mechanics, vol. 7, no. 3, pp. 234-239, 2007.

[23] Y. Zheng and K. Shimada, "Development of improved MCF rubber on MCF touch sensor," Journal of Japan Society Experimental Mechanics, vol. 8, no. 4, pp. 392-395, 2008.

[24] J. R. Pyne, "Conductive rubbers advance with new blacks," European Rubber Journal, pp. 17-20, 1981. 
[25] S. Wang and D. D. L. Chung, "Piezoresistivity in continuous carbon fiber polymer-matrix composite," Polymer Composites, vol. 21, no. 1, pp. 13-19, 2000.

[26] J. Cao, Q. Wang, and H. Dai, "Electromechanical properties of metallic, quasimetallic, and semiconducting carbon nanotubes under stretching," Physical Review Letters, vol. 90, no. 15, Article ID 157601, 4 pages, 2003.

[27] B. L. Pruitt, "Measurement system for low force and small displacement contacts," Journal of Microelectromechanical Systems, vol. 13, no. 2, pp. 220-229, 2004.

[28] G. Ausanio, A. C. Barone, C. Campana et al., "Giant resistivity change induced by strain in a composite of conducting particles in an elastomer matrix," Sensors and Actuators A, vol. 127, pp. 56-62, 2006.

[29] V. Maheshwari and R. F. Saraf, "High-resolution thin film device to sense texture by touch," Science, vol. 312, no. 9, pp. 1501-1504, 2006.

[30] R. D. Sherman, L. M. Middleman, and S. M. Jacobs, "Electron transport processes in conductor-filled polymers," Polymer Engineering Science, vol. 23, no. 1, pp. 36-46, 1983.

[31] P. Sheng, "Fluctuation-induced tunneling conduction in disordered materials," Physical Review B, vol. 21, no. 6, pp. 2180 2195, 1980.

[32] P. H. Meijer and A. V. Roggen, "Polymer electronic devices," Journal of Molecular Electronics, vol. 4, no. 2, pp. 119-124, 1988.

[33] K. Shimada, "Theoretical investigation by quantum mechanics on the tunnel diode effect of electric conductive characteristics and haptic sensing in MCF rubber," Journal of Sensors. In press.

[34] A. V. Roggen and P. H. E. Meijer, "The effect of electrodepolymer interfacial layers on polymer conduction," IEEE Transactions on Electrical Insulation, vol. EI-21, no. 3, pp. 307311, 1986.

[35] A. V. Roggen and P. H. E. Meijer, "The effect of electrodepolymer interfacial layers on polymer conduction,part 2," in Device Summary, Molecular Electronic Devices, F. L. Carter, R. E. Siatkowski, and H. Wohltjen, Eds., Elsevier, Amsterdam, The Netherlands, 1988.

[36] D. K. Ferry, Quantum Mechanics, An Introduction for Device Physics and Electrical Engineers, Springer, Tokyo, Japan, 2006, translated by: Y. Ochiai, M. Uchinami, K. Matsuda and K. Ishibashi. 

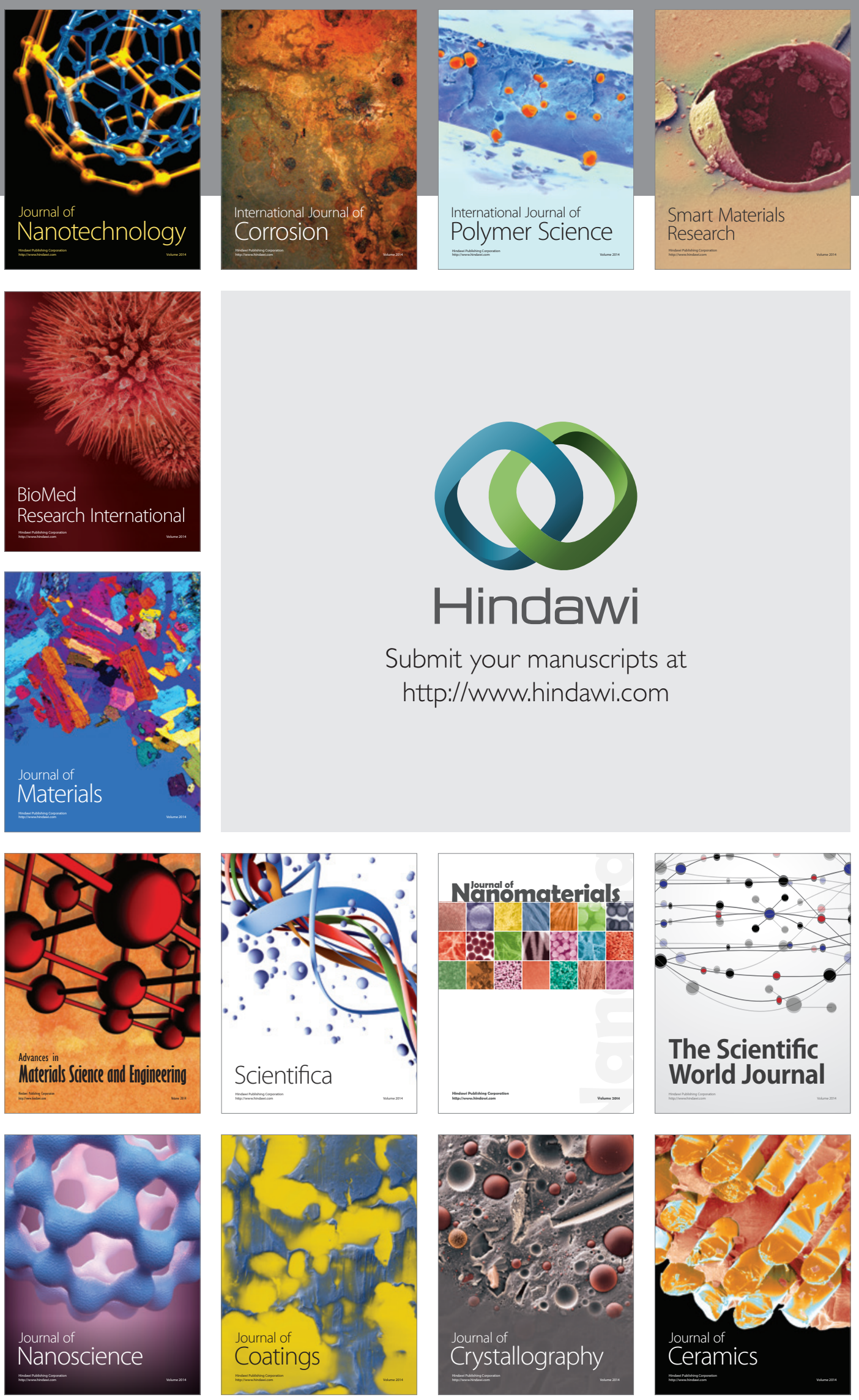

The Scientific World Journal

Submit your manuscripts at

http://www.hindawi.com

\section{World Journal}

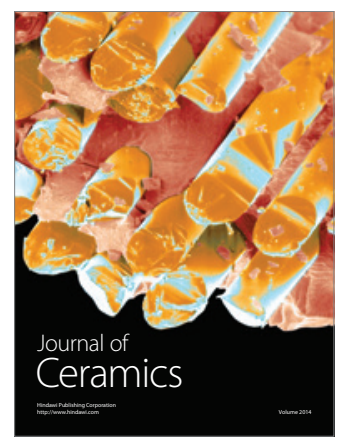

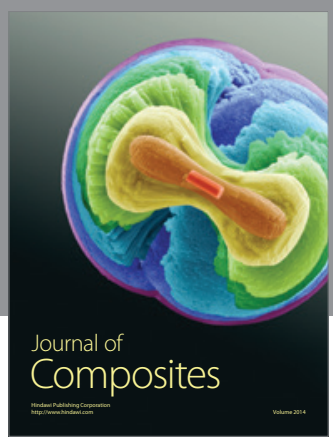
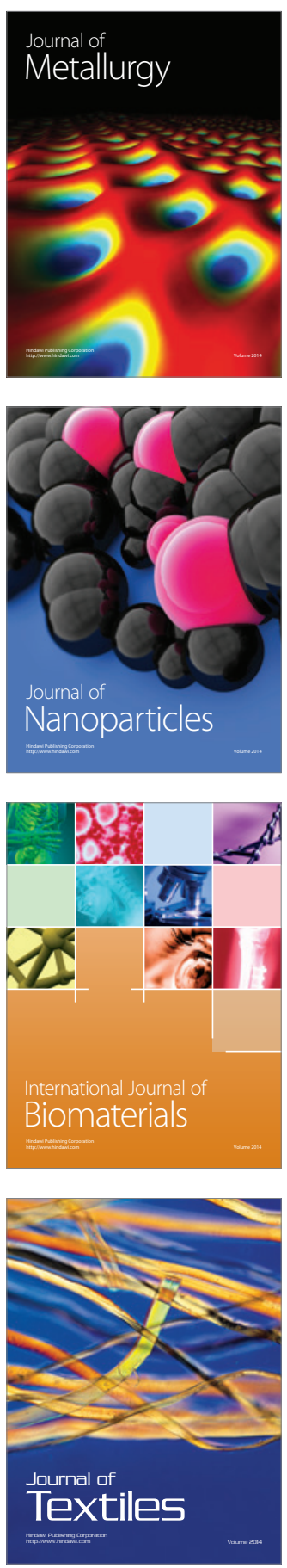